De la Fuente, A. y Gómez-Landero Rodríguez, L.A. (2019). Diferencias motoras en atletas cadetes de taekwondo según nivel competitivo / Motor Differences in Cadet Taekwondo Athletes According to Competition Level. Revista Internacional de Medicina y Ciencias de la Actividad $\begin{array}{llllllll}\text { Física y } & \text { el } & \text { Deporte } & \text { vol. } & 19 & \text { (73) } & \text { pp. } & 63-75\end{array}$ Http://cdeporte.rediris.es/revista/revista73/artdiferencias1002.htm DOI: http://doi.org/10.15366/rimcafd2019.73.005

\title{
ORIGINAL
}

\section{DIFERENCIAS MOTORAS EN ATLETAS CADETES DE TAEKWONDO SEGÚN NIVEL COMPETITIVO}

\section{MOTOR DIFFERENCES IN CADET TAEKWONDO ATHLETES ACCORDING TO COMPETITION LEVEL}

\author{
De la Fuente, A. ${ }^{1}$ y Gómez-Landero Rodríguez, L.A. ${ }^{2}$ \\ ${ }^{1}$ Club Deportivo CDE Sánchez Élez-Sanabria (España) ana.delafuente@hotmail.com \\ 2 Universidad Pablo de Olavide. Facultad del Deporte (España) lagomrod@upo.es
}

Código Unesco: 5899 Educación Física y Deporte / Physical Educaction; 5312.99 Deporte profesional / profesional sports.

Clasificación Consejo de Europa: 4. Educación física y deporte comparad / Physical Education and sport compared; 9. Cinantropometría / Kineanthropometry; 17. Otras: Rendimiento deportivo / Others: Sport performance

Recibido 6 de abril de 2017 Received April 6, 2017

Aceptado 2 de octubre de 2017 Accepted October 2, 2017

\section{RESUMEN}

El objetivo de este estudio es observar las diferencias entre atletas cadetes de Taekwondo de diferentes niveles competitivos en términos antropométricos, de potencia muscular, flexibilidad y velocidad de reacción. Veinte atletas varones fueron analizados, divididos en dos grupos: medallistas nacionales y no medallistas nacionales. Para cada atleta se tomaron datos antropométricos, de velocidad de reacción, potencia muscular y rango de movimiento articular de la articulación coxofemoral. Los atletas medallistas mostraron valores significativamente menores en altura, envergadura, tiempo de reacción óculo-podal, y tiempo de reacción óculo-manual con mano no dominante; $y$ valores mayores para longitud de piernas, flexión pasiva de cadera, y abducción de cadera tanto activa como pasiva. Las leves diferencias observadas entre grupos en potencia muscular, grasa corporal y flexión activa de cadera no concuerdan con estudios previos. Conocer está información podría ser de interés para los entrenadores para llevar a cabo programas de detección de talentos. 
PALABRAS CLAVE: Taekwondo, deporte de combate, rendimiento, detección de talentos, perfil competitivo.

\section{ABSTRACT}

The aim of this study was to determine the differences between Taekwondo cadet athletes belonging to different competition levels in terms of anthropometry, legs power, flexibility and reaction time. Twenty male athletes were analysed, divided in two groups: national medallist and non-national medallist. Data of every athlete's anthropometric measurements, reaction speed ability, leg power and hip ROM (range of movement) were taken. Medallist athletes showed significantly lower values for height, arm span, foot reaction time (dominant and non-dominant) and non-dominant hand reaction time; and higher values for leg length, passive hip flexion, active hip abduction and passive hip abduction in ROM tests. Slight differences observed between medallists and non-medallists in leg power (CMJ), body fat ( $\Sigma$ skinfolds) and active hip flexion do not agree with previous studies. Knowing this information could be interesting for coaches in order to carry out talent detection programs.

KEY WORDS: Taekwondo, combat sport, performance, talent detection, competitive profile.

\section{INTRODUCCIÓN}

Actualmente, el taekwondo (TKD) es un deporte olímpico de combate cuyos eventos competitivos están regulados a nivel regional, nacional e internacional por categorías según edad, sexo y peso corporal de los atletas. De acuerdo con la normativa de la Federación Mundial de Taekwondo (World Taekwondo Federation, 2016), para ganar un combate los competidores buscan obtener más puntos que su oponente ejecutando técnicas válidas de patada y puño en las áreas de puntuación permitida, o por knockouttécnico (abandono del rival), durante un enfrentamiento de tres asaltos de dos minutos, con un minuto de descanso entre los mismos.

En cualquier modalidad deportiva, la identificación de talentos es esencial para la temprana especialización y entrenamiento de los atletas. Por ello, determinar los factores de rendimiento específicos del deporte es necesario en inicio para el proceso de detección de talentos. En TKD, la categoría cadete (atletas desde 12 a 14 años) es la primera categoría de edad contemplada internacionalmente en competiciones oficiales (World Taekwondo Federation, 2016). Los campeonatos de esta categoría de edad tienen la misma estructura y características que los campeonatos de categorías superiores, por lo que es una etapa sensible para la identificación de talentos.

Se han encontrado muy pocas referencias en la literatura científica sobre los factores de rendimiento en atletas de categoría cadete. Las medidas antropométricas, como la altura o la longitud de las extremidades, han sido identificadas como una variable de rendimiento en TKD en general, de acuerdo 
con la idea de que unos miembros superiores más largos permiten cubrir una mayor área con un menor gasto energético, en las acciones de bloqueo en las que se defienden las zonas de puntuación (tronco y cabeza), y unos miembros inferiores más largos se traducen en una palanca más larga para generar mayor potencia en las técnicas ofensivas de patada (Kazemi et al., 2006; Markovic et al., 2005). Otros factores antropométricos como la composición corporal son también decisivos en TKD, puesto que las categorías competitivas están divididas por peso, y los atletas buscan tener el mínimo porcentaje de masa grasa en beneficio de la masa corporal para optimizar la ratio potencia-peso corporal (Bridge et al., 2014). La naturaleza balística de las técnicas de patada requiere elevada capacidad explosiva de los miembros inferiores, especialmente de la musculatura extensora (Kazemi et al., 2006; Suzana \& Pieter, 2009). La flexibilidad, definida como el rango de movimiento (ROM) de una articulación, es una habilidad particularmente necesaria en TKD debido a que las técnicas de patada, especialmente aquellas dirigidas al casco del adversario, requieren un gran ROM de la articulación de la cadera para su ejecución (Bridge et al., 2014; Suzana \& Pieter, 2009). El tiempo de reacción es también importante a la hora de conseguir puntos frente a un oponente, tanto para efectuar un golpeo exitoso cuando aparece una oportunidad como para bloquear una acción ofensiva del rival.

La importancia de estas variables para el éxito en competición ha sido demostrada en atletas junior y senior (Bridge et al., 2014; Gaeini et al., 2009; Kim et al., 2010; Plon et al., 2014). En cuanto a composición corporal, estudios previos realizados por Sadowski et al. (2005), Kazemi et al. (2006), Markovic et al. (2005), y Pieter (2008) muestran un menor porcentaje graso en los atletas con mejores resultados competitivos, todos ellos realizados con sujetos de categorías junior y senior. También ha sido demostrado, aunque pobremente, que una mayor longitud de miembros inferiores podría suponer un mejor rendimiento competitivo en TKD (Kazemi et al., 2006; Sadowski et al., 2012). De acuerdo con investigaciones previas, así como con estudios en disciplinas deportivas similares como karate o artes marciales mixtas (Balsalobre et al., 2012; Marinho et al., 2011), algunos estudios como el de Markovic et al. (2005), Casolino et al., (2012) y Chiodo et al. (2011) han aportado que los atletas senior de nivel internacional en TKD tenían mayor fuerza explosiva de miembros inferiores (medida con salto con contramovimiento, CMJ) que atletas senior de nivel sólo nacional. Los estudios que han evaluado la flexibilidad utilizando el test Sit and Reach han concluido que a pesar de que es una variable que puede ser importante en el rendimiento en TKD, no es una variable que distinga atletas de diferentes niveles de éxito deportivo (Bridge et al., 2014; Kazemi et al., 2006). Sin embargo, se necesitan más investigaciones utilizando test angulares de ROM, más cercanos a la acción deportiva (Bridge et al., 2014). Atendiendo al tiempo de reacción, Fontani et al. (2006) en un estudio con karatekas observaron un tiempo significativamente menor en atletas senior del equipo nacional en comparación con atletas senior de nivel recreativo, datos que quizá podrían ser similares en TKD por las características cercanas de ambas disciplinas. Se precisan más estudios con atletas en edades tempranas para determinar aquellas características que predicen el rendimiento competitivo en las primeras categorías, pero todo apunta a que serán determinantes todas aquellas capacidades que también lo son en edades posteriores (junior y senior). 
Considerando todos estos factores, el objetivo de este estudio es comparar los datos obtenidos de las variables de antropometría, potencia de piernas, flexibilidad (ROM) y tiempo de reacción en atletas de categoría cadete de TKD, para observar si existen diferencias entre los valores obtenidos por atletas de diferente nivel competitivo, y comprobando así si éstas variables podrían ser determinantes para el éxito competitivo de acuerdo con los resultados en atletas junior y senior.

\section{MATERIALES Y MÉTODOS}

\section{Participantes}

Veinte atletas de TKD de edades comprendidas entre los 14-15 años (todos ellos nacidos en el mismo año natural) fueron estudiados justo al terminar su etapa competitiva en categoría cadete. Todos los sujetos tenían más de 4 años de experiencia en campeonatos oficiales. Los atletas que componen la muestra de este estudio son miembros de la selección regional de la Comunidad de Madrid y clubes adscritos a la misma, resultando ser todos los competidores de la modalidad de combate existentes con esta edad y en esta región en el momento de la investigación. De acuerdo con los resultados en campeonatos nacionales oficiales, los atletas fueron divididos en dos grupos: medallistas $(n=8)$ y no medallistas $(n=12)$. Todos los sujetos y sus tutores legales firmaron un consentimiento informado, mostrando su acuerdo con los procedimientos de estudio y la publicación de los datos obtenidos. El Comité Ético de Investigación Humana universitario proporcionó la aprobación para este estudio.

\section{Toma de datos}

Las medidas antropométricas se tomaron de acuerdo a las normas de la Sociedad Internacional para el Avance de la Cineantropometría (ISAK) (MarfellJones et al., 2006). Se tomaron datos de seis pliegues cutáneos: tricipital, subescapular, abdominal, suprailíaco, muslo y pierna. El sumatorio de los seis pliegues es la variable utilizada para el análisis estadístico de la investigación.

El tiempo de reacción se midió a través de una versión del test de reacción óculo-podal propuesto por Padaguan (2014), replicándolo para la medida del tiempo de reacción óculo-podal y modificándolo ligeramente para la el tiempo de reacción óculo-manual. Para ambos se utilizó el sistema de contacto Chronojump-Boscosystem $\AA$, conectando el controlador temporal del sistema (Chronopic 3) a un ordenador, y dos dispositivos de contacto ligados al mismo. Para el test de reacción óculo-manual (EHRT), los dispositivos de contacto conectados al Chronopic 3 fueron dos pulsadores, uno para el investigador y otro para el sujeto. Cuando el investigador soltaba el pulsador, la luz LED del Chronopic 3 se encendía como señal para que el sujeto accionase su pulsador en el menor tiempo posible. El atleta estaba sentado en una silla frente a una mesa, con su mano en la misma sobre una marca a 5,08 cm del pulsador. Se realizaron tres intentos de familiarización con un periodo de descanso entre ellos de 30 segundos, y después se procedió al test consistente en otras tres 
ejecuciones con 30 segundos de descanso entre las mismas. La media entre las tres ejecuciones es la variable empleada para el análisis estadístico de la investigación. Se realizó el mismo procedimiento para la otra mano. Si a lo largo del test alguna ejecución era técnicamente incorrecta, el sujeto debía repetirla.

Para el test de reacción óculo-podal (EFRT) se realizó el mismo procedimiento anterior, sustituyendo el pulsador del sujeto por una plataforma de contacto. El atleta estaba de pie con su pie ejecutor en una marca a $10,16 \mathrm{~cm}$ de la plataforma, manteniendo ambos talones pegados al suelo. Al percibir el estímulo visual (luz LED del Chronopic 3) activado por el investigador, el sujeto debía pisar con su pie ejecutor sobre la plataforma en el menor tiempo posible. Se realizaron de nuevo tres intentos de familiarización y tres ejecuciones del test con un periodo de descanso de 30 segundos entre todas ellas, y la media de las tres ejecuciones se utilizó como variable de análisis. Si alguna ejecución era técnicamente incorrecta, el sujeto debía repetirla.

Los datos para potencia de piernas se tomaron a través del salto con contramovimiento (CMJ), empleando la plataforma de contacto ChronojumpBoscosystem $\Theta$. Se realizaron dos ejecuciones de familiarización y tres válidas para el test, con 1 minuto de descanso entre cada una de ellas. Si alguna ejecución era técnicamente incorrecta, el sujeto debía repetirla. La altura máxima alcanzada en las tres ejecuciones se utilizó como variable de análisis.

Para los datos de ROM se emplearon dos pruebas: el test de elevación de pierna recta y la prueba de abducción desde $90^{\circ}$ de flexión. En ambas se tomaron medidas tanto del movimiento activo como pasivo, y de ambas piernas (dominante y no dominante). En el test de elevación de pierna recta, el sujeto debía estar en posición supina, piernas extendidas, con su tronco y su cabeza en contacto con el suelo, y levantar su pierna totalmente extendida realizando una flexión de cadera, hasta alcanzar la mayor altura posible. Para la prueba de abducción desde $90^{\circ}$ de flexión, el sujeto debía estar también en posición supina, en $90^{\circ}$ de flexión de cadera, con las piernas extendidas sobre una pared y la zona glútea en contacto con la misma, y abducir sus piernas lo máximo posible. Se colocaron pegatinas en los puntos anatómicos importantes: trocánter, maléolo externo y maléolo interno para el test de elevación de pierna recta; pubis y punto medio de la línea intermaleolar para la prueba de abducción desde $90^{\circ}$ de flexión. Se tomaron imágenes de cada ejecución con una cámara situada a 3 metros del sujeto; si alguna ejecución era técnicamente incorrecta, el sujeto debía repetirla. El ROM en ambos test se analizó con el software informático Kinovea $\Theta$, trazando ángulos utilizando los puntos anatómicos marcados.

\section{Procedimientos}

Los sujetos fueron evaluados en una sola sesión en la que se llevó a cabo toda la batería de test antropométricos y físicos de acuerdo a los protocolos descritos. La sesión comprendía aproximadamente una hora de duración, y un descanso de 10 minutos separaba cada prueba de la siguiente. Todos los participantes eran competidores activos en la modalidad de combate (única modalidad olímpica de TKD), y se encontraban en el primer trimestre de su etapa junior. Así, los atletas habían finalizado muy recientemente su etapa competitiva 
en categoría cadete y podía ser evaluado su rendimiento en ella de forma completa. Los participantes debían tener al menos 4 años de experiencia en competición, para garantizar que habían disputado campeonatos oficiales desde el inicio de la etapa cadete.

Al inicio de la sesión, los participantes fueron informados acerca del objetivo de estudio y el programa y desarrollo de las pruebas. Se había instado a los atletas a que evitaran cualquier actividad física en las 24 horas previas a la toma de datos, y no a participar en ninguna jornada competitiva al menos en los 3 días previos a la medición. Los datos fueron tomados personalmente por los autores en el siguiente orden: 1) medidas antropométricas (altura, envergadura, longitud de piernas y pliegues cutáneos -seis-); 2) EHRT; 3) EFRT; 4) CMJ; 5) test de ROM de cadera. Antes de las pruebas de reacción, los atletas realizaron un calentamiento estándar de 10 minutos de duración, que consistía en trote ligero y diferentes desplazamientos. Se pidió a los sujetos que no realizasen ningún ejercicio de estiramiento en este periodo. Antes del test CMJ, los evaluadores explicaron en detalle la técnica de ejecución del salto, y los participantes realizaron un calentamiento específico para este test, consistente en 2 series de diez saltos CMJ, con 1 minuto de descanso entre series. Antes de las pruebas de ROM de cadera, los atletas fueron instruidos para realizar otro calentamiento específico, consistente en 10 minutos de estiramientos y ejercicios de movilidad articular de la coxofemoral.

\section{Análisis estadístico}

Los datos obtenidos en cada variable fueron comparados entre ambos grupos: medallistas y no medallistas nacionales. Para la comparación entre los valores de variables paramétricas se empleó la prueba T de Student para el análisis de la distribución muestras independientes. El test $U$ de Mann-Whitney fue el utilizado para las variables de características no paramétricas. El nivel de significación fue determinado al $t=0,5$. Los datos se muestran con estadísticos descriptivos, media \pm desviación típica.

La comparación de datos entre el número de tomas de los pliegues y de ejecuciones de los test de reacción fue realizada por análisis de varianza de medidas repetidas propuesto por Hopkins (2000a; 2000b), utilizando el coeficiente de correlación intraclase $(\mathrm{CCl})$ para los pliegues cutáneos y el coeficiente de variación (CV) para los datos de tiempo e reacción. Todos los valores de $\mathrm{CCI}$ para los pliegues cutáneos fueron mayores de 0,98 , lo que indica que las medidas tienen una fiabilidad muy alta de acuerdo con lo expuesto por Vincent (1999). Todos los valores del CV para los datos de tiempo de reacción fueron menores a $7,1 \%$, lo que significa que las medidas son muy fiables de acuerdo con Atkinson et al. (1999).

\section{RESULTADOS}

Las principales relaciones significativas $(p<0,05)$ obtenidas con las pruebas $T$ de Student y $U$ de Mann-Whitney para las variables de estudio se muestran en la Tabla 1. 
Tabla 1. Comparación de las variables de estudio según nivel competitivo.

\begin{tabular}{|c|c|c|c|}
\hline Variable & Medallistas & No-medallistas & $\begin{array}{l}\text { Tamaño del } \\
\text { efecto }(d)\end{array}$ \\
\hline Alturat $^{t}$ & $163,188 \pm 6,12$ & $168,958 \pm 4,83^{*}$ & $-1,074$ \\
\hline Envergadurat & $163,250 \pm 5,23$ & $172,833 \pm 6,95^{\star}$ & $-1,558$ \\
\hline Longitud de piernas (absoluta) ${ }^{t}$ & $84,438 \pm 5,90$ & $83,667 \pm 4,00$ & 0,153 \\
\hline Longitud de piernas $(\% \text { altura total })^{t}$ & $51,388 \pm 2,19$ & $49,507 \pm 1,53^{*}$ & 0,996 \\
\hline$\Sigma$ pliegues cutáneosu & $53,549 \pm 20,06$ & $52,468 \pm 26,93$ & 0,046 \\
\hline $\mathrm{CMJ}^{\mathrm{t}}$ & $32,042 \pm 4,85$ & $27,185 \pm 8,01$ & 0,734 \\
\hline \multicolumn{4}{|l|}{ EHRT } \\
\hline Mano dominante ${ }^{t}$ & $0,386 \pm 0,02$ & $0,419 \pm 0,05$ & $-0,867$ \\
\hline Mano no dominante & $0,401 \pm 0,02$ & $0,451 \pm 0,06^{*}$ & $-1,118$ \\
\hline \multicolumn{4}{|l|}{ EFRT } \\
\hline Pie dominante ${ }^{t}$ & $0,422 \pm 0,02$ & $0,475 \pm 0,04^{*}$ & $-1,676$ \\
\hline Pie no dominante & $0,434 \pm 0,03$ & $0,507 \pm 0,04^{*}$ & $-2,065$ \\
\hline \multicolumn{4}{|l|}{ Elevación de pierna recta activa } \\
\hline Pierna dominante ${ }^{t}$ & $83,630 \pm 9,21$ & $73,750 \pm 15,27$ & 0,784 \\
\hline Pierna no dominante ${ }^{t}$ & $77,000 \pm 11,19$ & $67,080 \pm 17,17$ & 0,685 \\
\hline Media entre ambas piernas ${ }^{t}$ & $80,313 \pm 9,79$ & $71,250 \pm 14,80$ & 0,722 \\
\hline \multicolumn{4}{|l|}{ Elevación de pierna recta pasiva } \\
\hline Pierna dominante ${ }^{u}$ & $115,250 \pm 15,25$ & $90,920 \pm 20,50^{*}$ & 1,347 \\
\hline Pierna no dominante ${ }^{t}$ & $110,250 \pm 16,20$ & $84,330 \pm 20,96^{\star}$ & 1,384 \\
\hline Media entre ambas piernas ${ }^{t}$ & $112,75 \pm 15,59$ & $87,625 \pm 20,58^{\star}$ & 1,376 \\
\hline \multicolumn{4}{|l|}{ Abducción } \\
\hline Activa $^{t}$ & $143,630 \pm 13,77$ & $120,580 \pm 11,50^{\star}$ & 1,817 \\
\hline Pasivat $^{t}$ & $160,250 \pm 15,21$ & $130,167 \pm 13,65^{\star}$ & 2,082 \\
\hline
\end{tabular}

Las variables marcadas con * presentan diferencias significativas entre grupos.

$\mathrm{V}^{\mathrm{t}}$ indica que la comparación entre variables se realizó mediante el test T de Student, y vu indica que la comparación entre grupos se realizó con el test U de Mann-Whitney.

Se observan diferencias significativas en las variables antropométricas altura, envergadura y longitud relativa de piernas (Tabla 1). El tiempo de reacción óculo-podal es también significativamente diferente entre el grupo de medallistas y el de no medallistas; en los valores de reacción óculo-manual se observaron diferencias únicamente en la ejecución con mano no dominante, a pesar de que la media entre ambas manos es claramente menor (Tabla 1). Los valores de la variable ROM de la articulación coxofemoral presentan también diferencias significativas entre ambos grupos, en el rango de flexión en su variante pasiva con ambas piernas y en abducción tanto activa como pasiva con ambas piernas (Tabla 1). No se observaron diferencias entre grupos para las variables de salto (CMJ), grasa corporal ( $\Sigma$ pliegues cutáneos) y flexión activa de la articulación coxofemoral (test de elevación de pierna recta) (Tabla 1). 


\section{DISCUSIÓN}

El principal objetivo de este estudio es comparar datos de variables de antropometría, potencia de miembros inferiores, flexibilidad y tiempo de reacción en atletas de TKD de categoría cadete, para observar si existen diferencias entre los valores obtenidos sujetos de diferente nivel de éxito competitivo, y comprobando así si dichas variables son determinantes para el rendimiento en TKD en esa etapa de edad. Para conocimiento del lector, este es el primer estudio que evalúa variables físicas en atletas de categoría cadete.

Considerando que el TKD es un deporte en el que las técnicas de patada constituyen la forma de ataque predominante, las características antropométricas pueden jugar un rol relevante en la distancia desde la que el atleta será capaz de golpear. Se han encontrado diferencias significativas entre grupos en las variables antropométricas, pero los resultados para las variables altura y envergadura son contrarios a la idea aportada por otros autores (Kazemi et al., 2006; Markovic et al., 2005), mostrando valores menores los sujetos medallistas de nuestro estudio que los no medallistas (Tabla 1). Sin embargo, nuestros datos sí concuerdan con los presentados por Cular et al. (2011) para deportistas senior, y en el caso de esta investigación quizá podrían ser explicados por la individualidad de los factores de desarrollo en la pubertad, en la que los cambios corporales que tienen lugar (como crecer en altura) hacen que el atleta necesite un tiempo de adaptación a sus nuevas estructuras (RuizFernandez, 2006). Esto sugiere que aquellos atletas que no han experimentado todavía esos cambios de altura están mejor adaptados a sus propias características corporales y serían más eficientes en competición, compensando la menor altura con otras capacidades como mayor velocidad y agilidad (Cular et al., 2011). Por otro lado, la poca muestra del presente estudio puede ser la culpable de estos resultados contradictorios.

Se encontraron también diferencias significativas entre grupos para la variable longitud relativa de piernas, mostrando valores más altos los medallistas, pero la diferencia no fue tan marcada para la variable longitud de piernas (absoluta). Estos datos sugieren que un prototipo de atleta con miembros inferiores largos en relación al resto de su cuerpo puede resultar ventajoso, en concordancia con el hecho de que unas piernas más largas proporcionan una distancia más larga desde la que poder golpear al oponente, y una palanca mayor con la que ejercer mayor potencia con la patada (Bridge et al., 2014; Kazemi et al., 2006; Markovic et al., 2005).

Las diferencias significativas obtenidas para la variable tiempo de reacción, que muestran que los medallistas reaccionan con mayor la velocidad que los no medallistas ante un estímulo visual, concuerdan con los resultados obtenidos por otros autores en estudios con atletas de categoría senior, tanto de TKD como de modalidades similares (Coskun et al., 2014; Fontani et al., 2006). El posible carácter predictivo de rendimiento competitivo de esta variable podría explicarse porque un menor tiempo de reacción proporciona una respuesta más rápida del atleta a las acciones del oponente, siendo así capaz de bloquearlas o esquivarlas para evitar ser puntuado. En acciones ofensivas también podría considerarse una variable determinante, puesto que cuanto más rápida sea la 
ejecución de una técnica, mayor probabilidad de que el atleta aproveche la ocasión que se presenta para puntuar. La velocidad de reacción mejora con la práctica (Coskun et al., 2014), de manera que estos datos quizá muestran una relación recíproca entre el tiempo de reacción y los resultados competitivos: un menor tiempo de reacción podría ayudar a los atletas a ejecutar sus acciones con mayor probabilidad de éxito y, con ello, a ganar mayor número de combates. De esta forma, si ganan combates, pasarán más rondas y su tiempo de práctica será mayor, teniendo más oportunidades de mejorar su capacidad de reacción que los atletas que pierden en primera ronda. Las investigaciones en este campo demuestran que el tiempo de reacción tiene una gran variabilidad en función de la edad y la maduración biológica individual (Coskun et al., 2014). Por ello, se precisan más estudios en categorías de edades tempranas, puesto que los datos de atletas senior tendrán muy poca transferencia a los valores mostrados por atletas cadetes.

Comparando el tiempo de reacción óculo-manual y óculo-podal, nuestros datos concuerdan con los aportados por Pfister et al. (2014) con sujetos no deportistas, siendo menor el tiempo de reacción con la extremidad superior.

El ROM de la articulación coxofemoral ha sido ampliamente estudiado por otros autores en atletas junior y senior, y ha sido descrito como una capacidad determinante para atletas de TKD en tanto en cuanto permite ejecutar técnicas de patada con el mejor recorrido y amplitud (Bridge et al., 2014; Sadowski et al., 2012; Suzana y Pieter, 2009). La utilización del test Sit and Reach para evaluar el ROM de cadera en muchas de estas investigaciones no permite asegurar la participación únicamente del movimiento de esta articulación, puesto que la influencia de la longitud de las extremidades y la movilidad vertebral ha sido demostrada como sesgo de la prueba (Arregui y Matínez de Haro, 2001). Por esta razón en el presente estudio se han utilizado pruebas angulares, de forma que los valores obtenidos correspondan sólo a la participación coxofemoral (Bridge et al., 2014; Ayala et al., 2012; López Miñarro et al., 2012; Luque Suárez et al., 2010). Se han encontrado diferencias significativas entre ambos grupos de atletas cadetes en los test de ROM de cadera, en las variables flexión pasiva (tanto pierna dominante como no dominante) y abducción activa y pasiva. Los valores más elevados de los sujetos medallistas concuerdan con los aportados para atletas junior y senior (Bridge et al., 2014; Sadowski et al., 2012; Suzana y Pieter, 2009), pero difieren con la afirmación de que la flexibilidad parece no discriminar entre deportistas de TKD de diferente nivel competitivo (Bridge et al., 2014; Kazemi et al., 2006). Sin embargo, la influencia de factores externos a la articulación coxofemoral en el test Sit and Reach, ampliamente empleado en estas investigaciones, podrían restar valor a los resultados de estos estudios. Se necesitan más datos en este campo utilizando tests angulares para evaluar el ROM de la articulación coxofemoral, y así poder comparar datos de diferentes edades y determinar fiablemente si esta capacidad resulta diferenciadora entre medallistas y no medallistas de todas las categorías.

La flexión activa de cadera no presenta diferencias significativas entre ambos grupos, aunque los valores sí son ligeramente superiores en medallistas que en no medallistas. Esta falta de significación podría explicarse por factores como el tamaño muestral o los diferentes cambios biológicos de los atletas, 
susceptibles en esta etapa madurativa a presentar mucha variación individual y por tanto datos dispersos. Otros estudios con deportistas senior sugieren que la no significatividad de estas diferencias podría explicarse porque la flexión activa de cadera es una variable considerada como básica para un competidor de TKD, a la hora de ejecutar técnicas de patada (Bridge et al., 2014; Casolino et al., 2012). Por ello, la naturaleza balística de las patadas en una situación real de competición difiere de las características activas simples del test de ROM utilizado en este estudio, que no presenta componente balístico y por tanto no se relaciona completamente con el gesto competitivo. La imposibilidad de medir la flexibilidad balística es una limitación de esta investigación, siendo quizá la forma de flexibilidad más relacionada con las acciones reales competitivas.

La potencia de miembros inferiores no mostró diferencias significativas entre ambos grupos de diferente nivel competitivo. A pesar de que no exista significación, los valores obtenidos por los atletas sí son ligeramente superiores en medallistas frente a no medallistas, y el tamaño del efecto es considerado moderado-alto (Chiodo et al., 2011), por lo que quizá tamaño muestral o los diferentes cambios madurativos de los atletas podrían haber acercado estas diferencias. De la misma forma que para la flexión activa de cadera, estudios previos mostraron que la potencia de piernas es una variable considerada básica para el TKD, a la hora de ejecutar técnicas de patada que superen el umbral de potencia requerido por el sistema de puntuación electrónico oficial para puntuar en cada categoría (World Taekwondo Federation, 2016; Casolino et al., 2012). Esta suposición de la potencia de piernas como capacidad básica podría explicar que esta variable no sea discriminatoria entre atletas de diferente nivel competitivo. Además, ha sido demostrado que los atletas de categoría senior obtienen valores más altos en CMJ que los deportistas junior (Bridge et al., 2014), lo que refleja la marcada variación en la composición de las fibras musculares y la activación de las unidades motoras en función de la edad, en relación con el desarrollo biológico y madurativo de los deportistas (Komi y Karlsson, 1978). Esta continua evolución a lo largo de las transiciones de edad podría ser una razón que explique los resultados tan pobres en atletas de categoría cadete, que presentarían unas características musculares aún poco maduras para convertirse en diferenciadoras.

De acuerdo con las medidas realizadas en este estudio, las variables antropométricas (altura, envergadura y longitud relativa de piernas), el tiempo de reacción óculo-podal, el tiempo de reacción óculo-manual con la mano no dominante, ROM en flexión pasiva de la articulación coxofemoral, y ROM en abducción activa y pasiva de ambas piernas son factores significativamente diferenciadores del nivel competitivo en atletas cadetes de TKD.

\section{CONCLUSIONES}

Este estudio pretende aportar los primeros datos como posible guía para futuras investigaciones sobre atletas de TKD de categoría cadete y sus características competitivas. Esta necesidad se sustenta en el hecho de que esta categoría es la primera etapa de edad internacionalmente reconocida para competiciones oficiales en TKD, y existen muy pocas referencias en la bibliografía de la modalidad con deportistas de esta edad que puedan ser 
comparadas con categorías superiores. Los resultados obtenidos señalan que existen diferencias significativas para las variables antropométricas (altura, envergadura y longitud relativa de piernas), el tiempo de reacción óculo-podal, el tiempo de reacción óculo-manual con la mano no dominante, ROM en flexión pasiva de la articulación coxofemoral, y ROM en abducción activa y pasiva de ambas piernas entre los valores obtenidos por atletas medallistas nacionales y no medallistas. Por tanto, los hallazgos de la presente investigación sugieren que las variables antropométricas, la velocidad de reacción y el ROM de la articulación coxofemoral podrían ser utilizados en programas de detección de talentos y seleccionar así deportistas con una mayor predisposición al éxito competitivo.

La principal limitación de este estudio es la dificultad para encontrar suficiente muestra de las características específicas necesarias para este tipo de investigación (edad, experiencia competitiva), lo que obliga a interpretar y extrapolar los resultados con cautela. Se necesitan más estudios con atletas de categoría cadete, incluyendo un mayor tamaño muestral, para poder obtener resultados más generalizables a la población de esta edad. Algunos factores como la flexibilidad balística, que puede ser importante en la competición de $T K D$, requieren ser investigadas en el futuro.

De acuerdo con los resultados obtenidos, los entrenadores podrían evaluar estas características determinantes de rendimiento en sus atletas, con el objetivo de proporcionarles un entrenamiento de tecnificación tan pronto como sea posible. Los hallazgos obtenidos en esta investigación deberían intentar ser replicados en estudios futuros, para observar así si estas variables muestran diferencias en otras poblaciones de la misma etapa de edad, y con ello poder aportar recomendaciones específicas para el entrenamiento de tecnificación de TKD en categoría cadete.

\section{REFERENCES}

Arregui, J.A., \& Martínez De Haro, V. (2001). Estado actual de las investigaciones sobre la flexibilidad en la adolescencia. Rev int Med Cienc Act Fis Deporte, 2(1).

Atkinson, G., Nevill, A.M., \& Edwards, B. (1999). What is an acceptable amount of measurement error? The application of meaningful 'analytical goals' to the reliability of sports science measurtements made on a ratio scale. In: Communications to the Annual Conference of the British Association of Sport and Exercise Sciences (BASES); J Sports Sci, 17(1): 18.

Ayala, F., Sainz de Baranda, P., Cejudo, A., \& Santonja, F. (2012). Pruebas angulares de estimación de la flexibilidad isquiosural: análisis de fiabilidad y validez. Rev Andal Med Deporte, 5(2), 67-74. DOI: https://doi.org/10.1016/S1888-7546(12)70011-4

Balsalobre-Fernández, C., Martínez-Majolero, V., Villacieros-Rodríguez, J., \& Tejero-González, C.M. (2012). Diferencias en el salto vertical y la velocidad de patada mae-geri entre karatekas internacionales y nacionales. Rev Artes Marciales Asiát, 8(1): 13-20. DOI: https://doi.org/10.18002/rama.v8i1.835 
Bridge, C.A., Ferreira da Silva Santos, J., Chaabène, H., Pieter, W., \& Franchini, E. (2014). Physical and Physiological Profiles of Taekwondo Athletes. Sports Med, 44: 713-733. DOI: https://doi.org/10.1007/s40279-014$\underline{0159-9}$

Casolino, E., Cortis, C., Lupo, C., Chiodo, S., Minganti, C., \& Capranica, L. (2012). Physiological versus Psychological evaluation in Taekwondo elite athletes. Int $J$ Sports Physiol Perform, 7, 322-331. DOI: https://doi.org/10.1123/ijspp.7.4.322

Chiodo, S., Tessitore, A., Cortis, C., Lupo, C., Ammendolia, A., Iona, T., \& Capranica, L. (2011). Effects of official Taekwondo competitions on allout performances of elite athletes. J Strength Cond Res, 25:334-9. DOI: https://doi.org/10.1519/JSC.0b013e3182027288

Coskun, B., Koçak, S., \& Saritas, N. (2014). The comparison of reaction times of karate athletes according to age, gender and status. OUA, Series Physical Education and Sport / Science, Movement and Health, 14(2), 97-101.

Cular, D., Krstulovic, S., \& Tomljanovic, M. (2011). The differences between medallists and non-medallists at the 2008 Olympic Games Taekwondo tournament. Hum Mov, 12(2): 165-170. DOI: https://doi.org/10.2478/v10038-011-0015-9

Fontani, G., Lodi, L., Felici, A., Migliorini, S., \& Corradeschi, F. (2006). Attention in athletes of high and low experienced engaged in different open skill sport. Percept Motor skill, 102: 791-805.

Gaeini, A.A., Fallahi, A.A., Kazemi, M., Mahmodi, Y., \& Moradyan, K. (2009). Defining the relationship between anthropometric, physiological and body composition characteristics of male elite Tae Kwon Do athletes with their success. Copenhagen: Daekyung Books, 2009.

Hopkins, W.G. (2000a). Measures of Reliability in Sports Medicine and Science. Sports Med, 30(1), 1-15. Available at http://www.sportsci.org/resource/stats/; accessed on 23.07.2016.

Hopkins, W.G. (2000b). Reliability from consecutive pairs of trials (Excel spreadsheet). A new view of statistics. sportsci.org: Internet Society for Sport Science. Available at http://www.sportsci.org/resource/stats/xrely.xls; accessed on 28.07.2016.

Kazemi, M., Waalen, J., Morgan, C., \& White, A.R. (2006). A profile of Olympic Taekwondo Competitors. J Sports Sci Med, 114-121.

Kim, J.W., Kwon, M.S., Yenuga, S.S., \& Kwon, Y.H. (2010). The effects of target distance on pivot hip, trunk, pelvis, and kicking leg kinematics in Taekwondo roundhouse kicks. Sports biomech, 9(2): 98-114. DOI: https://doi.org/10.1080/14763141003799459

Komi, P.V., \& Karlsson, J. (1978). Skeletal muscle fibre types, enzyme activities and physical performance in young males and females. Acta Physiol Scand, 103, 210-218. DOI: https://doi.org/10.1111/j.17481716.1978.tb06208.x

López-Miñarro, P.A., Vaquero-Cristóbal, R., Muyor, J.M., Alacid, F., \& Isorna, M. (2012). Validez de criterio del test sit-and-reach como medida de la extensibilidad isquiosural en piragüistas. $C C D, 7(20), 95-101$. DOl: https://doi.org/10.12800/ccd.v7i20.55 
Luque-Suárez, A., Fuente-Hervías, M.T., Barón-López, F.J., \& LabajosManzanares, M.T. (2010). Relación entre el test de elevación de pierna recta y el test ángulo poplíteo en la medición de la extensibilidad isquiosural. Fisioterapia, 32(6), 256-263. DOI: https://doi.org/10.1016/j.ft.2010.07.004

Marfell-Jones, M., Olds, T., Stewart, A., \& Carter, L. (2006). International standards for anthropometric assessment. Potchefstroom: ISAK.

Marinho, B.F., Del Vecchio, F.V., \& Franchini, E. (2011). Condición física y perfil antropométrico de atletas de artes marciales mixtas. Rev Artes Marciales Asiát, 6(2), 7-18.

Markovic, G., Misigoj-Durakovic, M., \& Trninic, S. (2005). Fitness profile of elite Croatian female taekwondo athletes. Coll Antropol, 29(1), 93-99.

Padaguan, J. (2014). Reliability of an eye-foot reaction time test in ChronojumpBoscosystem. Sport SPA, 11(2), 15-18.

Pfister, M., Lue, J.C.L., Stefanini, F.R., Falabella, P., Dustin, L., Koss, M.J., \& Humayun, M.S. (2014). Comparison of reaction response time between and foot controlled devices in simulated microsurgical testing. BioMed Res Int, available at http://dx.doi.org/10.1155/2014/769296; accessed on 23.09.2016.

Pieter, W. (2008). Body build of elite junior taekwondo athletes. AKUT, 13: 99106. DOI: https://doi.org/10.12697/akut.2008.13.08

Plon, J., Fransen, J., Lenoir, M., \& Segersm, V. (2014). The value of non-sportspecific characteristics for talent orientation in young male judo, karate and taekwondo athletes. Arch Budo, 10: 147-154.

Ruiz-Fernández, M.L. (2006). The sports medicine in the child. AMD, 13(114): 305-310.

Sadowski, J., Gierczuk, D., Miller, J., Cieslinski, I., \& Buszta, M. (2012). Success factors in male WTF taekwondo juniors. JCSMA, 1, 47-51. DOI: https://doi.org/10.5604/20815735.1047647

Suzana, M.A. \& Pieter, W. (2009). Motor ability profile of junior and senior Taekwondo club athletes. Braz J Biomotricity, 3(4), 353-331.

Vincent, W.J. (1999). Statistics in kinesiology. Champaign (IL): Human Kinetics.

World Taekwondo Federation, WTF (2016). Competition Rules and Interpretation. Available at http://www.worldtaekwondofederation.net/rules/ accessed on 19.12.2016.

Número de citas totales / Total references: 30 (100\%) Número de citas propias de la revista/ Journal's own references: $1(3,33 \%)$

Rev.int.med.cienc.act.fís.deporte - vol. 19 - número 73 - ISSN: 1577-0354 